\title{
First Demonstration of Cladding Pumped Few-moded EDFA for Mode Division Multiplexed Transmission
}

\author{
E-L Lim, Y. Jung, Q. Kang, T. C. May-Smith, N.H.-L. Wong, R. Standish, \\ F. Poletti, J.K. Sahu, S. Alam and D.J. Richardson \\ Optoelectronics Research Centre, University of Southampton, Southampton, SO17 1BJ, UK. \\ Authore-mail address:ell@orc.soton.ac.uk
}

\begin{abstract}
We report the first experimental demonstration of a cladding pumped FM-EDFA supporting 4 mode groups. The modal gains are measured to be $>20 \mathrm{~dB}$ between $1540 \mathrm{~nm}-1570 \mathrm{~nm}$ with a modal differential gain of $\sim 4 \mathrm{~dB}$ among the mode groups.

OCIS codes: (060.2320) Fiber optics amplifiers and oscillators; (060.2330) Fiber optics communications.
\end{abstract}

\section{Introduction}

Mode division multiplexing (MDM) for high-speed long-haul transmission is currently under intense investigation as a means to overcome the capacity limit of single-mode systems. To realize the energy and cost savings offered by MDM systems, the individual guided modes should be simultaneously amplified within a few-mode erbium doped fiber amplifier (FM-EDFA) [1]. To date, FM-EDFAs that simultaneously amplify 4 linearly polarized (LP) mode groups have been demonstrated based on a core-pumping scheme [2]. However, as the number of modes is scaledup significantly further then it will be challenging to meet the associated power requirements from single-mode diodes. Moreover, even if this is technically possible, multiplexing a number of single-mode pump diodes together to generate sufficient pump power is an expensive way of pumping such an amplifier. Cladding pumping represents a possible way to address these issues. High power, low cost (in terms of $\$ / \mathrm{W}$ ) multimode pump diodes operating in the multi-watt regime are now readily available now given the emergence and success of the high power cladding pumped fiber laser [3]. Cladding pumping has previously been shown as a viable approach to pumping both multicore [4] and multi-element amplifiers [5] but as of yet has not been demonstrated for a few-mode amplifier. Herein, we address this issue and demonstrate for the first time the feasibility of a cladding pumped FM-EDFA amplifying all the spatial modes of an erbium doped fiber supporting 4 mode groups.

\section{Experiment setup}

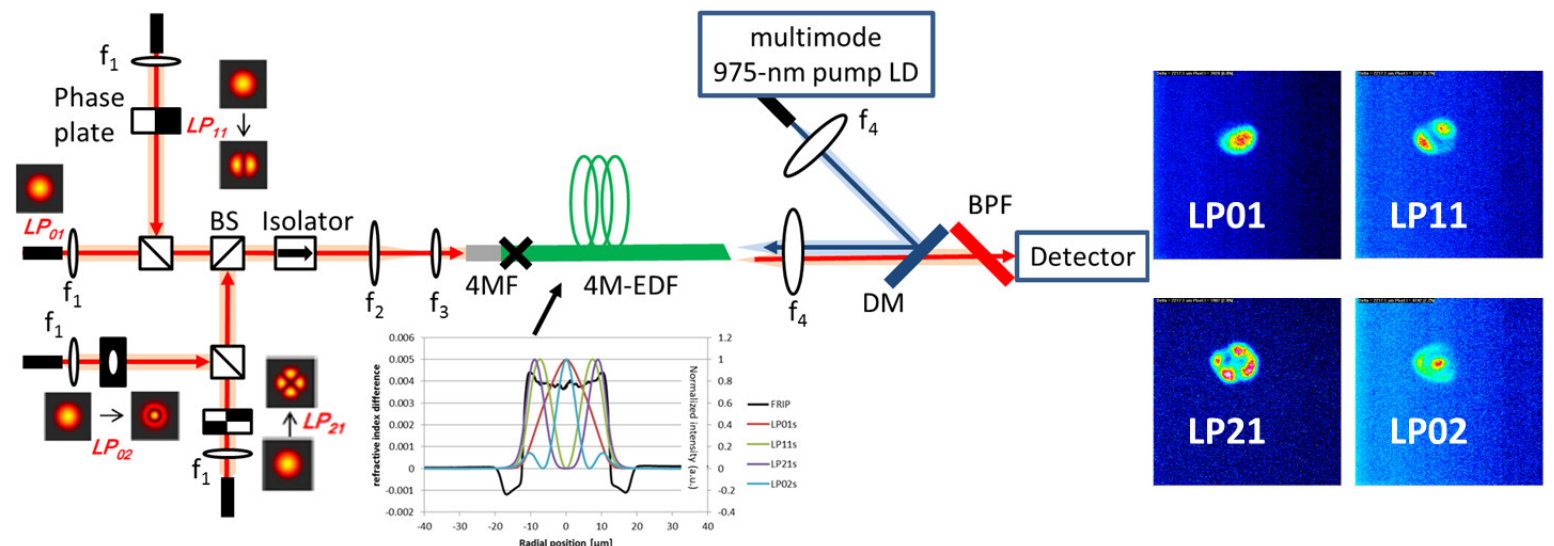

Fig. 1. Schematic diagram of the 4-moded cladding pumped erbium doped fiber amplifier (4M-EDFA). BS: non-polarizing beam splitter, DM: dichroic mirror, 4MF: passive 4-moded fiber, 4M-EDF: 4-moded erbium doped fiber, f1, f2 and f3: lenses with focal lengths of 4.5, 125 and $3.1 \mathrm{~mm}$ respectively. The CCD camera images show the mode profile after amplification.

Fig.1 shows the schematic diagram of our cladding pumped 4-moded EDFA (CP 4M-EDFA) that simultaneously amplifies the $\mathrm{LP}_{01}, \mathrm{LP}_{11}, \mathrm{LP}_{21}$ and $\mathrm{LP}_{02}$ mode groups. A mode multiplexer based on a collection of phase plates and beam splitters was used to selectively excite the individual mode group in a 4 mode group passive fiber (4MF) using four tunable external cavity lasers. The $4 \mathrm{MF}$ was then spliced directly to a cladding pumped 4 mode group erbium doped fiber (4M-EDF). The splice losses between the 4MF and 4M-EDF for the different modes 
were measured as $0.5 \pm 0.3 \mathrm{~dB}$. The refractive index and the erbium doping profile of the EDF are similar to that of ref [1]. The primary difference between this fiber and that of [1] is that the current fiber has a low-index polymer coating to define the pump waveguide (NA 0.44). The 4M-EDF has an outer cladding diameter of $97 \mu \mathrm{m}$ and a core diameter of $\sim 26 \mu \mathrm{m}$. The estimated effective NA of the core is $\sim 0.10$. The estimated cladding absorption is $\sim 1.08 \mathrm{~dB} / \mathrm{m}$ at $975 \mathrm{~nm}$. The fiber was counter-directional pumped via a dichroic mirror which was highly reflective $(>99 \%)$ at the pump wavelength and highly transmissive $(\sim 98 \%)$ at the signal wavelength. The multimode pump module can deliver an output power of up to $\sim 10 \mathrm{~W}$ and was wavelength-stabilized with a volume Bragg grating (VBG) at $975 \mathrm{~nm}$. Even though, the pump wavelength is $3 \mathrm{~nm}$ shorter than the peak absorption wavelength of $\sim 978 \mathrm{~nm}$, our simulation predicts that this will only have a slight impact on the total pump absorption and hence the signal gain. The output end of the $4 \mathrm{M}-\mathrm{EDF}$ was angled-cleaved at $\sim 8^{\circ}$ to suppress any back-reflections. The coupling efficiency of the pump power into the inner cladding was measured to be $\sim 80 \%$. The modal gain was measured by choosing a unique wavelength for the mode under test (MUT). At the output of the 4M-EDFA, the MUT was de-multiplexed via a tunable narrow bandpass filter (full width at half maximum=2nm). The wavelength allocation of the individual modes is discussed below. We estimate a measurement error of $\pm 0.5 \mathrm{~dB}$ in to all of our gain measurements. The right hand side of Fig.1 shows the measured mode profiles when the individual modes are amplified separately by the 4M-EDFA.

\section{Experimental results}

We first investigated the CP FM-EDFA with a 9-m long 4M-EDF. Fig. 2(a) shows the modal gain measured by setting the MUT at $1555 \mathrm{~nm}$ while other modes were fixed at $1560 \mathrm{~nm}$. Since the EDF predominantly experiences homogenous broadening, we assume that the effect due to spectral hole burning caused by the $1560 \mathrm{~nm}$ signal to be minimal. We therefore believe that in this way the modal gain of the individual modes at $1555 \mathrm{~nm}$ can be measured with low uncertainty originating from the spectral gain dependence. The DMG at $1555 \mathrm{~nm}$ was measured to be $\sim 4 \mathrm{~dB}$ for $>20 \mathrm{~dB}$ modal gains. The optical power efficiency (i.e. the ratio of total output signal power to launched pump power) was $\sim 8.5 \%$ at launched pump power of $\sim 2.07 \mathrm{~W}$. At the launched pump power of $\sim 1.7 \mathrm{~W}$, Fig. 2(b) shows the measured modal gain as a function of input MUT signal power with the wavelength assignment the same as that of Fig. 2(a). The signal gains decrease linearly with an increase in signal power for all modes due to the amplifier being operated in the unsaturated gain regime. The DMG did not show much dependence on the input signal power and remains at $\sim 3 \mathrm{~dB}$ for all the input signal powers investigated.
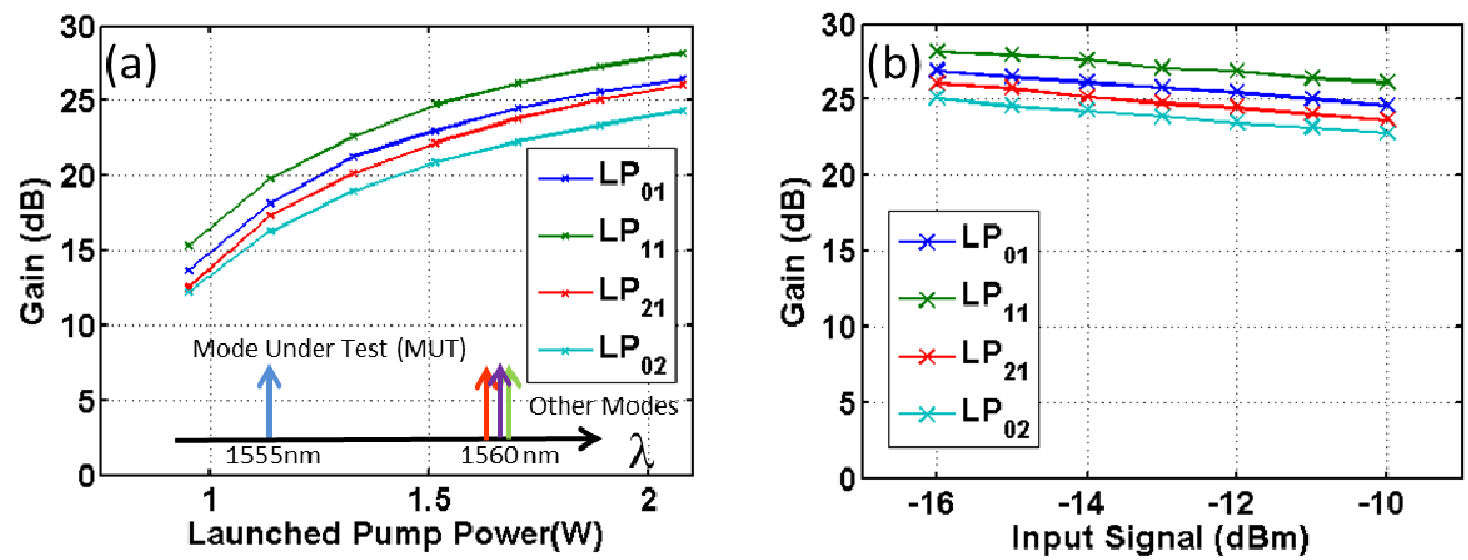

Fig.2. (a) Mode dependent gain as a function of input pump power for the four individual modes centered with the wavelength placement shown in the illustration. The input signal is $-10 \mathrm{dBm}$ for all modes (b) Measured modal gain as a function of input signal power per mode at launched pump power of $\sim 1.7 \mathrm{~W}$.

Fig. 3 shows the gain spectra of the various modes at a constant lauched pump power of $1.7 \mathrm{~W}$ for two different lengths of $4 \mathrm{M}-\mathrm{EDF}$, i.e. $9 \mathrm{~m}$ and $5 \mathrm{~m}$. The input signals of the various modes were fixed at $-11 \mathrm{dBm}$ for all wavelengths. The wavelength assignment of the various modes was such that, while the MUT was spectrally tuned, the other modes were fixed at $1560 \mathrm{~nm}$ (with the exception that when the MUT was centered at $1560 \mathrm{~nm}$, all other modes were shifted to $1555 \mathrm{~nm}$ ). Fig. 3(a) shows the gain spectra with a $9 \mathrm{~m}$ of EDF length. The gain peaked at $\sim 1565 \mathrm{~nm}$ and exhibited a $3 \mathrm{~dB}$ gain compression bandwidth of $\sim 15 \mathrm{~nm}$ spanning from $1560 \mathrm{~nm}$ to $1575 \mathrm{~nm}$. In order to shift the gain peak towards $1550 \mathrm{~nm}$ to better align with traditional C-band operation, we reduced the EDF length 
to 5m. As shown in Fig. 3(b), this resulted in a flatter gain spectra with a 3dB gain compression bandwidth increased to $\sim 30 \mathrm{~nm}$ spanning from $1540 \mathrm{~nm}$ to $1570 \mathrm{~nm}$. Although the reduction in the EDF length reduced the overall pump absorption to $\sim 5 \mathrm{~dB}$, the FM-EDFA still exhibited respectable modal gains of $\geq 20 \mathrm{~dB}$ between $1540 \mathrm{~nm}$ to $1570 \mathrm{~nm}$. Finally, we note that the noise figure of the 5m FM-EDFA was simulated to be $5-7 \mathrm{~dB}$ between $1540 \mathrm{~nm}$ to $1580 \mathrm{~nm}$ and will be measured in the future.
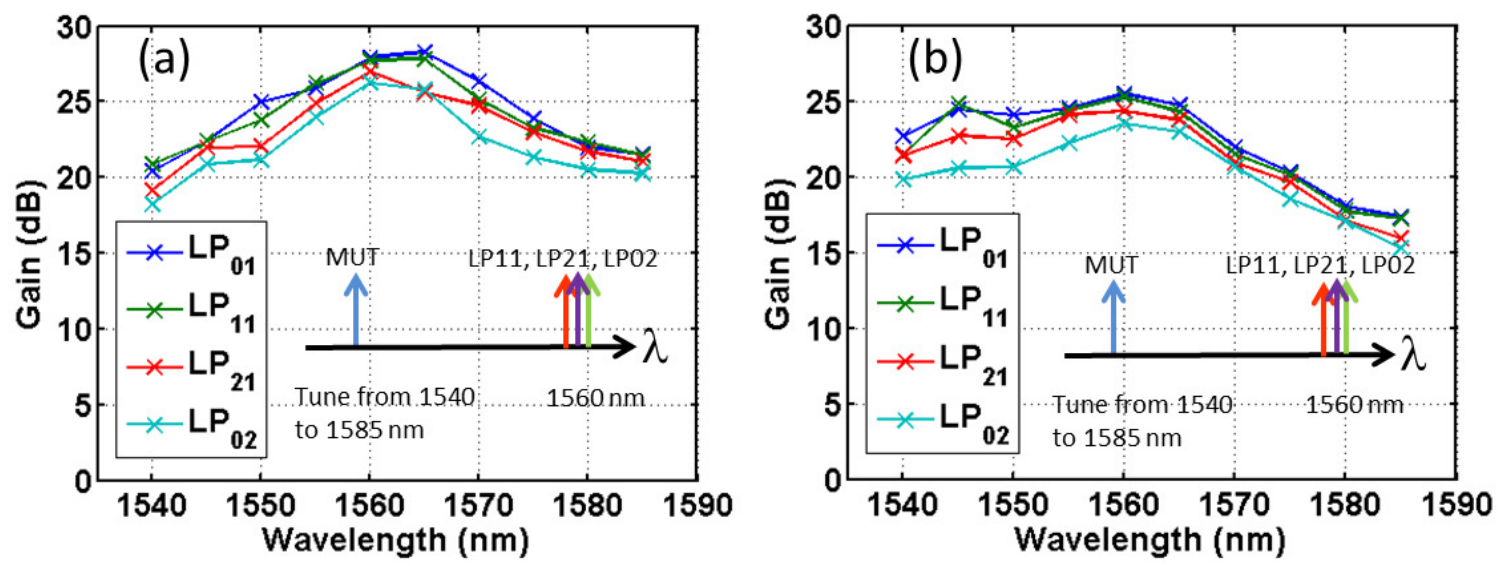

Fig.3. Gain spectra of various modes for a 4M-EDF with length of (a) $9 \mathrm{~m}$ and (b) $5 \mathrm{~m}$ at launched pump power of $\sim 1.7 \mathrm{~W}$..

\section{Conclusion}

We have demonstrated for the first time a cladding pumped few moded EDFA supporting 4-mode groups. The modal differential gain was determined to be $\sim 4 \mathrm{~dB}$ with $\mathrm{LP}_{11}$ exhibiting the highest modal gain. Reduction in the EDF length from $9 \mathrm{~m}$ to $5 \mathrm{~m}$ widens the operating window of the FM-EDFA from $15 \mathrm{~nm}$ to $30 \mathrm{~nm}$ and ensures near full C-band operation. We believe that the MDG can be reduced further by optimizing the core design whilst full Cband operation will be possible by optimizing the core-to-clad area ratio of the cladding pumped EDF.

This work was supported by the European Communities 7th Framework Program under grant agreement 258033 (MODE-GAP) and the UK EPSRC grant EP/J008591/1 (COMIMO) and

[1] Y. Jung, S. Alam, Z. Li, A. Dhar, D. Giles, I. Giles, J. Sahu, F. Poletti, L. Grüner-Nielsen, and D. Richardson, "First demonstration and detailed characterization of a multimode amplifier for space division multiplexed transmission systems," Opt. Express 19, B952-B957 (2011). [2] M. Salsi, D. Peyrot, G. Charlet, S. Bigo, R. Ryf, N. Fontaine, M. Mestre, S. Randel, X. Palou, C. Bolle, B. Guan, G. Le Cocq, L. Bigot, and Y. Quiquempois, "A Six-Mode Erbium-Doped Fiber Amplifier," in $38^{\text {th }}$ European Conference and Exhibition on Optical Communication, paper Th.3.A.6 (2012).

[3] D. Richardson, J. Nilsson, and W. Clarkson, "High power fiber lasers: current status and future perspectives [Invited]," J. Opt. Soc. Am. B 27, B63-B92 (2010).

[4] K. Abedin, T. Taunay, M. Fishteyn, D. DiGiovanni, V. Supradeepa, J. Fini, M. Yan, B. Zhu, E. Monberg, and F. Dimarcello, "Claddingpumped erbium-doped multicore fiber amplifier," Opt. Express 20, 20191-20200 (2012).

[5] V J F Rancano, S Jain, T C May-Smith, J K Sahu, P Petropoulos and D J Richardson, " First Demonstration of an Amplified Transmission Line Based on Multi-Element Fibre Technology," in $39^{\text {th }}$ European Conference and Exhibition on Optical Communication, paper PD1.C.2 (2013) 